\title{
The long-term outcomes of endoscopic third ventriculostomy in pediatric hydrocephalus, with an emphasis on future intellectual development and shunt dependency
}

\author{
Sukwoo Hong, MD, Daisuke Hirokawa, MD, Kenichi Usami, MD, PhD, and \\ Hideki Ogiwara, MD, PhD
}

Division of Neurosurgery, National Center for Child Health and Development, Tokyo, Japan

\begin{abstract}
OBJECTIVE The goal of this study was to clarify the long-term outcome of endoscopic third ventriculostomy (ETV) in pediatric hydrocephalus in light of the ETV Success Score (ETVSS), shunt dependency, and intellectual development.

METHODS The authors retrospectively analyzed pediatric patients with hydrocephalus who underwent ETV between 2002 and 2012 and who were followed for longer than 5 years as a single-center cohort. The data of the patients' pre- and postoperative status were collected. The relationships between ETVSS and the full-scale IQ as well as shunt dependency were analyzed. The usefulness of ETVSS for repeat ETV and the change of radiological parameters of ventricle size before and after ETV were also analyzed. The success of ETV was defined as no requirement for further CSF diversion procedures.
\end{abstract}

RESULTS Fifty ETVs were performed in 40 patients. The average ETVSS was 61 and the success rate at 6 months was $64 \%$. The mean follow-up was 9.9 years (5.2-15.3 years), and the long-term success rate of ETV was $50 \%$. The Kaplan-Meier survival curve continued to show a statistically significant difference among patients with a low, moderate, and high ETVSS, even after 6 months $(p=0.002)$. After 15 months from the initial ETV, no patients required additional CSF diversion surgery. There was no statistical significance between ETVSS and the long-term full-scale IQ or shunt dependency $(p=0.34$ and 0.12 , respectively). The radiological improvement in ventricle size was not associated with better future educational outcome.

CONCLUSIONS The ETVSS was correlated with the long-term success rate. After 15 months from the initial ETV, no patients required an additional CSF diversion procedure. The ETVSS was not considered to be correlated with long-term intellectual status.

https://thejns.org/doi/abs/10.3171/2018.7.PEDS18220

KEYWORDS ETV; ETVSS; Endoscopic Third Ventriculostomy Success Score; long-term follow-up; intellectual development; hydrocephalus

E NDOSCOPIC third ventriculostomy (ETV) is an effective treatment for hydrocephalus (HCP). The ETV Success Score (ETVSS) is a score used to predict the probability of ETV success at 6 months from the surgery. ${ }^{10}$ The ETVSS predicts the short-term success, but there have been few reports in the literature about the longterm outcome of ETV, especially after longer than 5 years. Published studies that have evaluated the relationship between the ETVSS and educational outcome have also been scarce. We performed a retrospective analysis to further clarify the long-term effects of ETV for pediatric HCP, including shunt dependency and intellectual development.

\section{Methods}

We analyzed patients who underwent ETV during the period between 2002 and 2012, and who were followed for at least 5 years. The exclusion criteria were those who were 18 years or older or who had choroid plexus coagulation performed in the same sitting, tumor resection within

ABBREVIATIONS ETV = endoscopic third ventriculostomy; ETVSS = ETV Success Score; FOHR = fronto-occipital horn ratio; $\triangle F O H R=$ change of FOHR; FSIQ = fullscale IQ; HCP = hydrocephalus; $m$ TVW = maximal third ventricle width; $\triangle \mathrm{mTVW}=$ change of $\mathrm{mTVW}$.

SUBMITTED April 22, 2018. ACCEPTED July 10, 2018.

INCLUDE WHEN CITING Published online October 12, 2018; DOI: 10.3171/2018.7.PEDS18220. 
TABLE 1. Baseline characteristics of 50 ETV cases in 40 patients

\begin{tabular}{|c|c|}
\hline Characteristic & No. \\
\hline \multicolumn{2}{|l|}{ Age } \\
\hline $0-30$ days & 1 \\
\hline $1-6$ mos & 10 \\
\hline $7-12$ mos & 16 \\
\hline $1-9$ yrs & 17 \\
\hline $10-17$ yrs & 6 \\
\hline $\operatorname{Sex}(M / F)$ & $23: 27$ \\
\hline \multicolumn{2}{|l|}{ Etiology of $\mathrm{HCP}$} \\
\hline Infection & 8 \\
\hline MMC & 1 \\
\hline IVH & 5 \\
\hline Nontectal tumor & 2 \\
\hline Aqueductal stenosis & 10 \\
\hline Tectal tumor & 9 \\
\hline Others & 15 \\
\hline Previous shunt (yes/no) & $12: 38$ \\
\hline \multicolumn{2}{|l|}{ ETVSS } \\
\hline $0-40$ & $11(22 \%)$ \\
\hline $50-70$ & $23(46 \%)$ \\
\hline $80-90$ & $16(32 \%)$ \\
\hline
\end{tabular}

$\mathrm{IVH}=$ intraventricular hemorrhage; $\mathrm{MMC}=$ myelomeningocele. ETVSS subgroups: low, 0-40; moderate, 50-70; high, 80-90.

6 months of ETV, or external ventricular drainage or shunt revision in the same sitting. We selected these exclusion criteria to evaluate the pure effect of ETV in the pediatric age group. We thought that tumor resection, choroid plexus coagulation, or external ventricular drainage were confounding factors for evaluation of the simple effect of ETV. We retrospectively analyzed patients' baseline characteristics, the ETVSS, subsequent surgeries due to worsening of HCP, current status of shunt dependency, and educational outcome. We defined the success of ETV as no additional CSF diversion surgeries for worsening of HCP. Based on the acquired data, we drew Kaplan-Meier curves. We classified the ETVSS as low (0-40), moderate (50-70), and high (80-90) and evaluated whether predictability of the ETVSS continues longer than 6 months. We classified the ETVSS into 3 subgroups as in previous studies.,12 KaplanMeier curves were drawn for 3 groups of the ETVSS (low, moderate, and high) and the $\mathrm{p}$ value was calculated using the log-rank test. We used the chi-square test to evaluate the adequacy of ETVSS for repeat ETV cases.

As for the educational outcome, we evaluated full-scale IQ (FSIQ) for some patients. For those whose FSIQ was not available, we dichotomized the patients into those who went to regular school and those who went to special school. We classified those with FSIQ of 75 or more as showing normal intellectual development, because 75 was the cutoff for percentile. FSIQ was obtained by the Kyoto Scale of Psychological Development or the Wechsler Intelligence Scale for Children. The collected FSIQ and educational status were compared to ETVSS to find any statistical significance by the ANOVA test and chi-square test, respectively.
TABLE 2. Success rate of ETV based on the first, second, and third procedure

\begin{tabular}{cccc}
\hline ETV & $\begin{array}{c}\% \text { w/ Long-Term } \\
\text { Success (no.) }\end{array}$ & $\begin{array}{c}\% \text { w/ 6-Mo } \\
\text { Success (no.) }\end{array}$ & $\begin{array}{c}\text { Average } \\
\text { ETVSS }\end{array}$ \\
\hline 1st op & $58(23 / 40)$ & $72(29 / 40)$ & 63 \\
\hline 2nd op & $25(2 / 8)$ & $38(3 / 8)$ & 53 \\
\hline 3rd op & $0(0 / 2)$ & $0(0 / 2)$ & 40 \\
\hline All ops & $50(25 / 50)$ & $64(32 / 50)$ & 61 \\
\hline
\end{tabular}

The overall 6-month success rate is similar to the average ETVSS.

As for the radiological parameters for ventricle size, the fronto-occipital horn ratio (FOHR) and maximal third ventricle width (mTVW) were calculated for the 3 time periods of preoperative, postoperative, and latest time. The change of FOHR $(\Delta \mathrm{FOHR})$ and change of mTVW $(\Delta \mathrm{mTVW})$ were calculated to evaluate if there was any difference between patients with normal intellectual development and those without. The Mann-Whitney U-test was used to analyze the association between radiological parameters of ventricle size and the current educational status in those in whom ETVs were successful. The Student t-test was used to analyze ETV cases irrespective of success or failure.

Data analyses were done using procedures as the sample in drawing Kaplan-Meier curves, evaluating ETVSS validity in the repeat ETV cases, and evaluating radiological parameters. Data analyses were done using patients as the sample in others. We used a p value of 0.05 as a cutoff to determine the statistical significance.

\section{Results}

Ninety ETVs in 80 patients were performed during 2002 to 2012. Of these, 40 patients with 40 ETVs were excluded (2 for concurrent choroid plexus coagulation, 3 for death within 6 months for causes other than HCP, 16 for concurrent ETV, 18 for tumor resection within 6 months, and 1 for concurrent shunt revision). We had 1 case (1.1\%) in which we could not fenestrate the third ventricle floor because it was thick, but this case was excluded for tumor resection within 6 months of ETV, after which no shunt was needed. The remaining 50 ETVs in 40 patients were identified, and the results are classified as low, moderate, and high scores in Table 1. ETVSS and the actual success rate are summarized in Table 2 . The long-term success rate was $58 \%(23 / 40), 25 \%(2 / 8)$, and $0 \%(0 / 2)$ in the first, second, and third ETV, respectively. The mean follow-up period was 9.9 years (range 5.2-15.3 years). The baseline characteristics are shown in Table 1. Figure 1 shows Kaplan-Meier curves for 3 subgroups of ETVSS; the $p$ value was 0.002 . The 3 curves did not cross each other or get close during the follow-up. Additional surgeries were performed in 25 cases (50\%) in total. Eighteen (36\%) ETVs failed within 6 months and another 7 (14\%) failed after 6 months from the most recent ETV. No ETVs failed after 15 months.

As for the status of shunt dependency, 29 of 40 patients (73\%) were shunt free. There was no statistical significance between ETVSS and the "final" status of shunt de- 


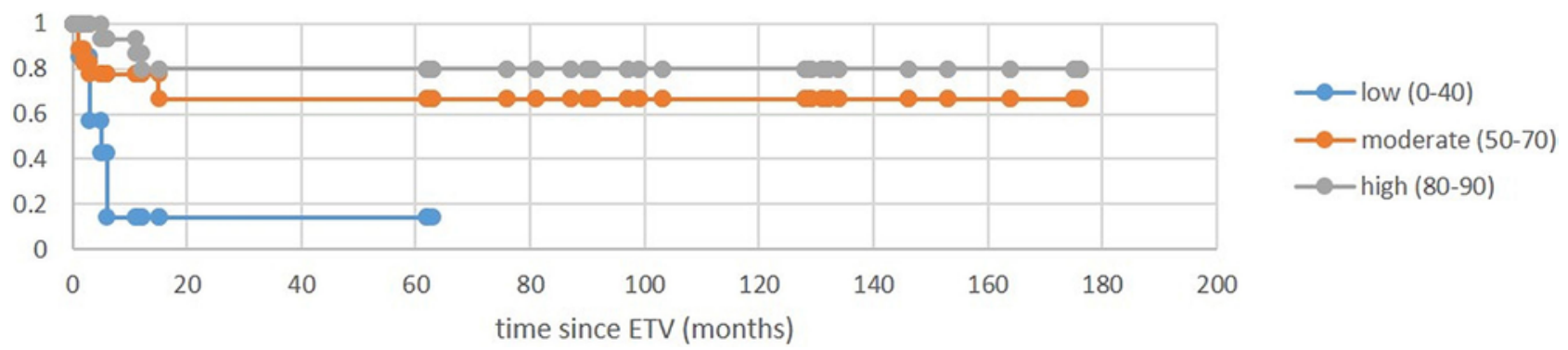

FIG. 1. Kaplan-Meier curves for resolution of HCP after ETV depending on the 3 subgroups $(p=0.002)$. Note that 7 cases recurred after 6 months but that no HCP recurred after 15 months from previous ETV. Figure is available in color online only.

pendency $(p=0.12)$. Table 3 shows the shunt status of each case of ETV performed. As for the educational outcome, the latest FSIQs were available for 19 patients. The average FSIQ was 91 (SD 19). The average FSIQs were 83, 88, and 101 in the low, moderate, and high ETVSS groups, respectively. There were no significant differences in FSIQ among those 3 groups $(p=0.34)$. The types of school (regular or special education) were available for all 40 patients (Table 4). There were 3 patients whose ETVSS subgroup changed when repeat ETV was performed (e.g., ETVSS of 70 [moderate group] at the first ETV and 80 [high group] at the second ETV). We allocated them into the subgroup of the last ETV. There were no significant differences in types of education among those 3 groups either $(p=0.48)$.

For those who underwent multiple ETVs, 8 patients underwent a second ETV, and 4 of these patients were shunt free and attending regular school at the final follow-up. On the other hand, 2 patients underwent a third ETV and turned out to be shunt dependent (one patient attending regular class, the other one in special classes). In total, there were 10 repeat ETV cases (Table 2), of which 6-month success was achieved in 0 of 5,1 of 3 , and 2 of 2 cases in the low, moderate, and high ETVSS subgroups, respectively. The $\mathrm{p}$ value for those proportions of success and failure was 0.007 . When 10 repeat ETV cases were combined with 40 single ETV cases, the $p$ value for the proportion of success and failure in each subgroup was 0.0005 .

As for the radiological parameters of ventricle size, we analyzed 24 cases with ETV success and 13 cases with ETV failure. For those with successful ETVs, there was no statistical significance between $\triangle F O H R$ and $\triangle \mathrm{mTVW}$ during the preoperative and postoperative time periods and the latest follow-up ( $\mathrm{U}$ value of 22-25 according to the Mann-Whitney U-test, critical value of 14). As for the

TABLE 3. ETVSS and the latest status of shunt dependency

\begin{tabular}{rccc}
\hline ETVSS & $\begin{array}{c}\text { Shunt Independent, } \\
\mathrm{n}=29\end{array}$ & $\begin{array}{c}\text { Shunt Dependent, } \\
\mathrm{n}=11\end{array}$ & $\begin{array}{c}\text { Total, } \\
\mathrm{N}=40\end{array}$ \\
\hline $0-40$ & $3(43)$ & $4(57)$ & 7 \\
\hline $50-70$ & $14(74)$ & $5(26)$ & 19 \\
\hline $80-90$ & $12(86)$ & $2(14)$ & 14 \\
\hline
\end{tabular}

$p=0.53$ for the comparison. The numbers in parentheses represent the percentage with each shunt status according to the total for that ETVSS subgroup. educational status, there was no statistical significance in $\triangle F O H R$ and $\Delta$ mTVW during the 3 time periods (Table 5 ). The mean time intervals between pre- and postoperative imaging, and between preoperative and latest imaging were 17 days and 88 months, respectively.

\section{Discussion}

Our overall ETV success rate was 50\% (58\% in the first ETV), lower than the average ETVSS of 63. However, ETV success at 6 months was $64 \%$. Because the ETVSS was originally devised to predict the 6-month outcome, it was also considered to be valid in our study (Table 2). And to make our study more externally valid, the overall HCP resolution curve of Fig. 1 follows a similar curve in a larger study done by Warf et al.$^{18}$ Our Fig. 1 demonstrates that ETVSS prediction of the success rate persists even after 6 months. On the other hand, our study showed that ETVSS did not predict the future status of shunt dependency or education (Tables 3 and 4). Also, the radiological parameters, such as $\triangle F O H R$ and $\triangle \mathrm{mTVW}$, were not associated with future educational status (Table 5).

\section{ETV Failure}

Considering the result that $72 \%$ of failures $(18 / 25)$ occurred within 6 months, ETV failed even after 6 months, and no HCP recurred after 15 months, we suggest that follow-up should be closely scheduled in the first 6 months, regularly in the next year, and with longer intervals thereafter. However, we must be aware that there is a small but real possibility that ETV could fail even after 10 years. ${ }^{2}$ Mobbs et al. suggested that placing a CSF reservoir for every ETV

\section{TABLE 4. ETVSS and the long-term postoperative educational} status

\begin{tabular}{cccc}
\hline ETVSS & Regular School & Special School & Total \\
\hline $0-40$ & $4(67)$ & $2(33)$ & 6 \\
\hline $50-70^{*}$ & $13.5(71)$ & $5.5(29)$ & 19 \\
\hline $80-90$ & $13(87)$ & $2(13)$ & 15 \\
\hline Total $^{*}$ & $30.5(76)$ & $9.5(24)$ & 40 \\
\hline
\end{tabular}

$p=0.48$ for the comparison. The numbers in parentheses represent the percentage with each shunt status according to the total for that ETVSS subgroup.

* One patient was getting both regular and special education, so we assigned 0.5 to both regular and special education groups. 
TABLE 5. Comparison between educational status and the mean radiological change in patients who went to regular school versus special school

\begin{tabular}{lllc}
\hline \multicolumn{1}{c}{ Mean Value } & $\begin{array}{c}\text { Regular } \\
\text { School }\end{array}$ & $\begin{array}{c}\text { Special } \\
\text { School }\end{array}$ & $\begin{array}{c}\text { p Value } \\
\text { (Student t-test) }\end{array}$ \\
\hline$\Delta$ FOHR (preop to postop) & -0.02 & -0.05 & 0.51 \\
\hline$\Delta$ FOHR (preop to latest) & -0.11 & -0.08 & 0.39 \\
\hline$\Delta$ mTVW (preop to postop) & -4 & -2.1 & 0.39 \\
\hline$\Delta$ mTVW (preop to latest) & -6.7 & -3.2 & 0.23 \\
\hline
\end{tabular}

surgery helps in case a late failure occurs,${ }^{14}$ which we practice, too. Seven patients (18\%) benefited from this practice-we had 7 late failures. Whether ETVSS adequately predicts the success rate of repeat ETV was evaluated by Breimer et al., who performed a retrospective analysis using a Dutch population to conclude the adequacy. ${ }^{2} \mathrm{~A}$ similar result was found in our Japanese population, suggesting the generalizability of the study. Vulcu et al. said that the shunt history at the time of ETV did not appear to have an impact on the long-term success of ETV. ${ }^{16}$ On the other hand, our study showed the statistical significance of shunt history on the long-term success of ETV $(p=0.0009)$. This may be partly due to the difference of ages. The subjects in the study published by Vulcu et al. were mostly adults (74\%), whereas our cohort consists of a pediatric population. Given that the ETVSS was originally devised based on the data of patients younger than 20 years, ${ }^{10}$ a different statistical outcome may result if the subjects were mostly adults.

\section{ETVSS and Future Shunt Status}

As for the status of shunt dependency, more than $70 \%$ of patients were shunt free. Even in the group with a low ETVSS, the rate of shunt independence was $43 \%$ (3/7). Considering this rate, it may be acceptable to perform ETV as a first-line treatment for pediatric HCP. It is counterintuitive that the future shunt status does not follow the trend of Fig. 1; this may be attributed to the small number of patients. With more subjects, we may have a statistically significant result based on the 3 divisions of the ETVSS.

\section{ETVSS and Future Educational Status}

Regarding the educational outcome, there was no statistical significance between ETVSS and the "final" educational status or the FSIQ ( $\mathrm{p}=0.48$ and 0.34 , respectively). Similar to the "final" status of shunt dependency, even if the ETVSS is low, it is worth proceeding with ETV in regard to intelligence (Table 5). Although it is true that those with a low ETVSS will more likely need to undergo repeat ETV or ventriculoperitoneal shunt placement in the future, it is worth trying to do at least $1 \mathrm{ETV}$ considering the fact that there is a meaningful proportion of patients who have attained normal intelligence in the low ETVSS subclass. There was no significant difference in the quality of life or neurocognition between ETV and shunting in the past studies. ${ }^{1,11,13,17}$ Hader et al. compared pre- and post-ETV intelligence scores to find that ETV was helpful in improving cognition. ${ }^{8}$ Also, several reports in the litera- ture suggest that ETV is worth trying before shunting even in those younger than 6 months. ${ }^{4,15}$

\section{Radiological Parameters Before and After ETV}

Several studies have suggested that the clinical success of ETV is not correlated with radiological ventricular size decrease. ${ }^{3,5,7}$ Furthermore, Kulkarni et al. found that large ventricle size after treatment was not correlated with neurocognitive injury in asymptomatic posttreatment patients with aqueductal stenosis. ${ }^{9}$ Our result that there was no statistical significance between educational status and the change in radiological parameters not only supports their finding, but also extrapolates that their finding is not exclusive to patients with aqueductal stenosis. Gianaris et al. showed 3 factors that may contribute to failure in those with a high ETVSS: preoperative lethargy, preoperative radiological finding of transependymal flow, and postoperative mTVW. ${ }^{6}$ We evaluated those 3 factors, but none showed statistical significance (data not shown). That may be due to the small number of subjects, so further larger studies might be needed to confirm those factors.

\section{Study Limitations}

There were several limitations in our study. First, it was retrospective and the number of subjects was not large. This may have led to the insignificant $\mathrm{p}$ value as to the future shunt status. Second, the objective value of intelligence, or FSIQ, was not available in most cases preoperatively, and in only approximately half of the postoperative cases, which may have made it difficult to appreciate the effect of ETV on the patient's future intelligence. Taking these limitations into account, it would be better to arrange the prospective studies of the larger sample size by evaluating the FSIQ in all patients with HCP before and after performing ETV.

\section{Conclusions}

The ETVSS was correlated with the long-term success rate. After 15 months from the initial ETV, no patients required an additional CSF diversion procedure. The ETVSS was not considered to be correlated with longterm educational status.

\section{References}

1. Azab WA, Mijalcic RM, Nakhi SB, Mohammad MH: Ventricular volume and neurocognitive outcome after endoscopic third ventriculostomy: is shunting a better option? A review. Childs Nerv Syst 32:775-780, 2016

2. Breimer GE, Dammers R, Woerdeman PA, Buis DR, Delye $\mathrm{H}$, Brusse-Keizer M, et al: Endoscopic third ventriculostomy and repeat endoscopic third ventriculostomy in pediatric patients: the Dutch experience. J Neurosurg Pediatr 20:314-323, 2017

3. Buxton N, Turner B, Ramli N, Vloeberghs M: Changes in third ventricular size with neuroendoscopic third ventriculostomy: a blinded study. J Neurol Neurosurg Psychiatry 72:385-387, 2002

4. Faggin R, Bernardo A, Stieg P, Perilongo G, d'Avella D: Hydrocephalus in infants less than six months of age: effectiveness of endoscopic third ventriculostomy. Eur J Pediatr Surg 19:216-219, 2009 
5. Garg A, Suri A, Chandra PS, Kumar R, Sharma BS, Mahapatra AK: Endoscopic third ventriculostomy: 5 years' experience at the All India Institute of Medical Sciences. Pediatr Neurosurg 45:1-5, 2009

6. Gianaris TJ, Nazar R, Middlebrook E, Gonda DD, Jea A, Fulkerson DH: Failure of ETV in patients with the highest ETV success scores. J Neurosurg Pediatr 20:225-231, 2017

7. Goumnerova LC, Frim DM: Treatment of hydrocephalus with third ventriculocisternostomy: outcome and CSF flow patterns. Pediatr Neurosurg 27:149-152, 1997

8. Hader WJ, Brooks BL, Partlo L, Hamilton M: Neuropsychological outcome after endoscopic third ventriculostomy. Can J Neurol Sci 41:729-734, 2014

9. Kulkarni AV, Donnelly R, Mabbott DJ, Widjaja E: Relationship between ventricular size, white matter injury, and neurocognition in children with stable, treated hydrocephalus. J Neurosurg Pediatr 16:267-274, 2015

10. Kulkarni AV, Drake JM, Mallucci CL, Sgouros S, Roth J, Constantini S: Endoscopic third ventriculostomy in the treatment of childhood hydrocephalus. J Pediatr 155:254-259, 259.e1, 2009

11. Kulkarni AV, Hui S, Shams I, Donnelly R: Quality of life in obstructive hydrocephalus: endoscopic third ventriculostomy compared to cerebrospinal fluid shunt. Childs Nerv Syst 26:75-79, 2010

12. Kulkarni AV, Riva-Cambrin J, Holubkov R, Browd SR, Cochrane DD, Drake JM, et al: Endoscopic third ventriculostomy in children: prospective, multicenter results from the Hydrocephalus Clinical Research Network. J Neurosurg Pediatr 18:423-429, 2016

13. Kulkarni AV, Shams I, Cochrane DD, McNeely PD: Quality of life after endoscopic third ventriculostomy and cerebrospinal fluid shunting: an adjusted multivariable analysis in a large cohort. J Neurosurg Pediatr 6:11-16, 2010

14. Mobbs RJ, Vonau M, Davies MA: Death after late failure of endoscopic third ventriculostomy: a potential solution. Neurosurgery 53:384-386, 2003
15. Ogiwara H, Dipatri AJ Jr, Alden TD, Bowman RM, Tomita T: Endoscopic third ventriculostomy for obstructive hydrocephalus in children younger than 6 months of age. Childs Nerv Syst 26:343-347, 2010

16. Vulcu S, Eickele L, Cinalli G, Wagner W, Oertel J: Longterm results of endoscopic third ventriculostomy: an outcome analysis. J Neurosurg 123:1456-1462, 2015

17. Warf B, Ondoma S, Kulkarni A, Donnelly R, Ampeire M, Akona J, et al: Neurocognitive outcome and ventricular volume in children with myelomeningocele treated for hydrocephalus in Uganda. J Neurosurg Pediatr 4:564-570, 2009

18. Warf BC, Mugamba J, Kulkarni AV: Endoscopic third ventriculostomy in the treatment of childhood hydrocephalus in Uganda: report of a scoring system that predicts success. J Neurosurg Pediatr 5:143-148, 2010

\section{Disclosures}

The authors report no conflict of interest concerning the materials or methods used in this study or the findings specified in this paper.

\section{Author Contributions}

Conception and design: Ogiwara, Hong. Acquisition of data: Hong, Hirokawa, Usami. Analysis and interpretation of data: Hong. Drafting the article: Hong. Critically revising the article: Ogiwara. Reviewed submitted version of manuscript: Ogiwara, Hirokawa, Usami. Approved the final version of the manuscript on behalf of all authors: Ogiwara. Statistical analysis: Hong.

\section{Correspondence}

Hideki Ogiwara: National Center for Child Health and Development, Tokyo, Japan. hideki_o@d5.dion.ne.jp. 